\title{
Alta recidiva y buenos resultados funcionales tras la resección artroscópica de la sinovitis villonodular pigmentaria de la rodilla
}

\section{- Autores.}

A. Isart ${ }^{1}$, P.E. Gelber ${ }^{1,2}$, M. Besalduch ${ }^{2}$, X. Pelfort ${ }^{1}$, J.I. Erquicia ${ }^{1}$, M. Tey-Pons ${ }^{1}$, J.C. Monllau ${ }^{1,3}$.

- Instituciones.

1- Institut Català de Traumatologia i Medicina de l'Esport (ICATME)-Hospital Universitari Quirón-Dexeus, Universitat Autònoma de Barcelona, Barcelona, España.

2- Servicio de Cirugía Ortopédica y Traumatología, Hospital de la Santa Creu i Sant Pau, Universitat Autònoma de Barcelona, Barcelona, España.

3- Servicio de Cirugía Ortopédica y Traumatología, Parc de Salut Mar, Universitat Autònoma de Barcelona, Barcelona, España.

\section{- Responsabilidades éticas:}

Protección de personas y animales. Los autores declaran que para esta investigación no se han realizado experimentos en seres humanos ni en animales.

Confidencialidad de los datos. Los autores declaran que en este artículo no aparecen datos de pacientes.

Derecho a la privacidad y consentimiento informado. Los autores declaran que en este artículo no aparecen datos de pacientes.

- Anna Isart Torruella. Teléfono.699852021. Email. isartanna@gmail.com C/Sabino de Arana, 5-19. 08028. Barcelona. España. 


\begin{abstract}
Alta recidiva y buenos resultados funcionales tras la resección artroscópica de la sinovitis villonodular pigmentaria de la rodilla
\end{abstract}

\title{
$\underline{\text { Resumen }}$
}

\section{$\underline{\text { Introducción }}$}

La sinovitis villonodular pigmentaria (SVP) es un trastorno de la proliferación sinovial de etiología incierta, con un tratamiento controvertido. El objetivo del estudio es valorar los resultados funcionales y la tasa de recurrencia en una serie de pacientes diagnosticados de SVP de rodilla, tanto en su forma difusa como en su forma localizada, tratados mediante resección artroscópica.

\section{Material y Métodos}

Estudio retrospectivo de 24 pacientes diagnosticados por resonancia magnética de SVP local/difusa entre 1996 y 2011. Se trataron 11 casos de forma localizada y 13 casos de forma difusa. Tras un seguimiento mediano de 60 meses (rango, 34-204). Se intervinieron mediante sinovectomia artroscópica y se valoraron funcionalmente en el postoperatorio con los tests IKDC, WOMET y Kujala.

\section{$\underline{\text { Resultados }}$}

8 pacientes afectados de SVP difusa recidivaron (61,5\% de las formas difusas). De ellos 2 requirieron radioterapia, 1 cirugía abierta por afectación extraarticular y 5 nueva resección artroscópica sin detectarse posteriormente nueva recidiva. En 6 pacientes se observaron lesiones asociadas ( 3 meniscopatía y 3 lesiones condrales). No hubo recidivas en la forma localizada. El IKDC mejoró de media 30,6 puntos, el WOMET 37,4 puntos y Kujala 34,03 puntos. 


\section{Discusión}

La SVP resecada mediante técnica artroscópica presenta buenos resultados funcionales y curativos a medio plazo con una baja morbilidad. La forma difusa de la SVP requiere con frecuencia una segunda intervención quirúrgica por su alta tasa de recidiva tras su resección artroscópica.

Palabras clave

Sinovitis villonodular, rodilla, artroscopia

\section{Abstract}

\section{Background}

Pigmented villonodular synovitis (PVS) is a synovial proliferation disorder of uncertain etiology, with certain controversys regardind its proper treatment. The purpose of the study was to evaluate the functional outcome and recurrence rate in a series of patients diagnosed of both the diffuse and the localized type of PVS and treated by arthroscopic resection.

\section{Methods}

Twenty-four patients diagnosed of PVS were retrospectively assessed. There were 11 cases with the diffuse type and 13 cases with the localized type of PVS. With a median follow-up of 60 months (range, 34-204). They underwent arthroscopic synovectomy. They were functionally evaluated with IKDC, WOMET and Kujala scores. 


\section{$\underline{\text { Results }}$}

8 out of 13 cases with the diffuse type of PVS recurred (61,5\%). Two of these patients were treated with radiation. One patient underwent surgical resection with an open procedure due to extraarticular involvement. The remaining 5 patients underwent a second arthroscopic resection, and no recurrence was subsequently observed. Cases with localized PVS did not recur after a single arthroscopic resection. IKDC, WOMET and Kujala scores improved 30,6, 37,4 and 34,03 points, respectively.

\section{Discussion}

Pigmented villonodular synovitis treated under arthroscopic resection showed good functional results at mid-term follow-up. A single arthroscopic resection was sufficient to treat the localized PVG, whereas the diffuse type of PVS required a second arthroscopic resection in most cases due to its high rate of recurrence.

Keywords

Villonodular synovitis, knee, arthroscopy. 


\section{INTRODUCCIÓN.}

La sinovitis villonodular pigmentaria (SVP) es una enfermedad de la membrana sinovial caracterizada por la proliferación de células mononucleares. Se trata de una patología infrecuente y benigna que afecta típicamente a la rodilla en adultos jóvenes $(<40 \text { años })^{1}$.

En la literatura se distinguen dos formas de presentación de la SVP, la difusa (SVPD) (Figura 1A) y la localizada (SVPL) (Figura 1B). La forma localizada se caracteriza por ser una lesión lobular y pendular de la sinovial, que suele presentarse en la región femoropatelar. Por el contrario, la SVPD se caracteriza por una afectación de toda o gran parte de la sinovial articular².

Si bien se considera de forma generalizada que el tratamiento de la SVP consiste en realizar una exéresis completa de la sinovial afectada, no existe consenso en cuanto a la técnica quirúrgica a utilizar; mientras algunos autores abogan por la resección mediante cirugía abierta ${ }^{1-6}$, otros describen similares tasas de recurrencia mediante una sinovectomía total artroscópica ${ }^{3,7-9}$. La sinovectomía artroscópica permite una mejor preservación de la funcionalidad de la rodilla, con pocas complicaciones y con una tasa de recurrencia aceptable, aunque esta última podría depender de la forma de SVP. Las terapias complementarias con radioterapia externa o con sinoviortesis isotópica han sido propuestas como alternativas en resecciones parciales o incompletas como tratamiento coadyuvante, con el objetivo de mejorar el control local de la enfermedad. No se ha documentado el éxito de una segunda resección quirúrgica ante una eventual recidiva de la SVP $2-14$. 
El objetivo del estudio fue valorar los resultados funcionales y la tasa de recurrencia de una serie de pacientes diagnosticados de SVP, tanto en su forma difusa como en su forma localizada, tratados mediante resección artroscópica. 


\section{MATERIAL Y MÉTODOS}

De una base de datos que incluían a todos los pacientes intervenidos de una rodilla mediante técnica artroscópica en nuestro centro, se seleccionaron aquellos diagnosticados de SVP entre los años 1996 y 2011.

Los criterios de inclusión en el trabajo fueron: 1) Pacientes con gonalgia; 2) Estudio preoperatorio completo con diagnostico presuntivo por resonancia magnética $(\mathrm{RM})$ de SVP; 3) Intervenidos mediante técnica artroscópica; 4) Con confirmación por parte de la anatomía patológica de SVP; 5) Con un seguimiento superior a los 24 meses.

Se excluyeron los pacientes con lesiones de SVP intraóseas.

Entre 1996 y 2011 se realizaron 6006 artroscopias de rodilla en nuestro centro, 24 de ellas cumplieron los criterios de inclusión en nuestro estudio (0,4\%). 6 casos quedaron excluidos $(0,1 \%)$ por lesiones intraóseas. El grupo de pacientes presentaba una mediana de seguimiento de 60 meses (rango, 34 - 204 meses) sin detectarse ningún abandono durante el seguimiento. Los resultados demográficos se hallan resumidos en la (Tabla 1). Se trataron 13 pacientes con forma difusa de SVP. Los 11 restantes afectados presentaban SVP localizada, todos ellos diagnosticados intraoperatoriamente. 7 de estos casos presentaban lesión en el compartimento posterior de la rodilla y en los 4 pacientes restantes, en el compartimento femoropatelar.

\section{Valoración funcional}

Se registraron en cada paciente el estado clínico y funcional tanto en el preoperatorio como en la última valoración del postoperatorio con los test IKDC ${ }^{15}$, 


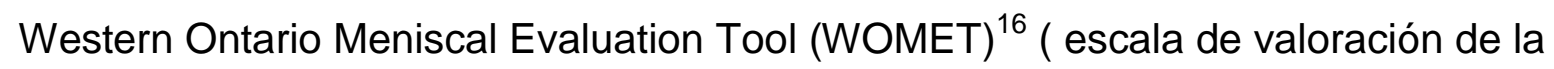

\section{Valoración radiográfica}

Se les realizó un estudio radiológico con radiografía telemétrica de las extremidades inferiores en carga, una radiografía de perfil, otra axial de rótulas y una proyección de Rosemberg (posteroanterior a 45ํ de flexión, en carga) de ambas rodillas en el preoperatorio, a los 6 meses y a los dos años en el postoperatorio, siguiendo siempre el mismo protocolo. Se utilizó la clasificación de Ahlbäck para estudiar el grado de artrosis, en las radiografías telemétricas preoperatorias y en el control de los 2 años postquirúrgicos. Asimismo se realizó una RM de la rodilla afectada con el fin de diagnosticar la lesión y su localización durante el preoperatorio (Figura 2).

\section{Técnica quirúrgica}

Todas las cirugías fueron realizadas por 4 cirujanos especializados en rodilla. Se llevaron a cabo de forma rutinaria 4 portales artroscópicos: dos anteriores (anterolateral y antero-medial) para acceder al compartimento femoropatelar y otros 2 posteriores (postero-lateral y postero-medial) y así acceder al compartimento posterior. Se efectuó una sinovectomía total con una terminal de 5,5mm incisional (Gator, ConMed Linvatec, Largo, FL, EU) en modo oscilante y se trataron las lesiones asociadas en caso que existiesen. Se obtuvo una biopsia de tejido sinovial en todos los casos, colocándose un drenaje intraarticular del número 10 durante las 
primeras 24 horas.

En el período postoperatorio se realizó profilaxis con heparina de bajo peso molecular durante un período de 15 días, siguiendo un protocolo de rehabilitación permitiendo la carga completa de la extremidad y de fortalecimiento muscular según tolerancia.

\section{Lesiones asociadas}

Seis $(25 \%)$ de los enfermos presentaron lesiones asociadas, diagnosticadas a través de la RM preoperatoria: 3 de ellos de meniscopatía y 3 de lesiones condrales. En el caso de las meniscopatías, afectaban al compartimento medial. Una fue tratada mediante sutura y las otras dos por medio de una meniscectomía parcial. En cuanto a las lesiones condrales dos eran de grado III y se les realizaron microfracturas y una tercera de grado IV a nivel de la tróclea no requirió ningún tratamiento. En los controles postoperatorios no se observaron ni empeoramiento de las lesiones asociadas ni tampoco cambios artrósicos en las radiografías.

\section{Anatomía patológica}

El análisis fue realizado por un anatomopatólogo experto en patología tumoral musculoesquelética. En todos los casos el diagnóstico fue realizado tras observarse la presencia de vellosidades y nódulos marrones formados por grupos de histiocitos con estroma fibroso, depósitos de hemosiderina, células gigantes y células espumosas ${ }^{7}$ en la muestra obtenida intraoperatoriamente.

\section{Análisis estadístico}


Para describir las variables categóricas se resumieron con frecuencias y porcentajes, mientras que en las variables cuantitativas se utilizaron la media y la desviación estándar o bien la mediana y percentiles 25 y 75 . Para analizar las diferencias pre y postoperatorias de los pacientes con los test IKDC, WOMET, Kujala y Tegner se utilizó el test no paramétrico de Wilcoxon, y para analizar las diferencias según la presencia de lesiones asociadas se utilizó el test U de Mann-Whitney. Se consideraron como estadísticamente significativos los valores de P inferiores a 0,05 y los análisis se llevaron a cabo con el programa estadístico SPSS 18.0 (SPSS inc., Chicago, IL). 


\section{RESULTADOS}

\section{Resultados funcionales}

Los resultados de los test funcionales evidenciaron una mejoría de 30,6 puntos en el $\operatorname{IKDC}(p=0,001), 37,4$ puntos en el WOMET $(p=0,001)$ y de 34,03 puntos en el Kujala $(p=0,001)$ (Tabla 2) en el global de los pacientes. Al dividir la muestra en pacientes afectos de SVPL y SVPD no se hallaron diferencias en ninguno de los test (Tabla 3). Al estudiar los resultados dividiéndolos en dos grupos (pacientes con SVP y pacientes con SVP más patología asociada) se encontró una mayor mejoría en el IKDC de 40,6 puntos $(p=0,05)$ y en el KUJALA de 46,8 puntos $(p=0,026)$ en los que presentaban patologías concomitantes (Tabla 4).

No hubo déficit de extensión en ningún caso, observándose una flexión final media de $127^{\circ}$ (rango, $\left.120^{\circ}-140^{\circ}\right)$. No se evidenciaron complicaciones peroperatorias ni postoperatorias en relación a la técnica quirúrgica, como trombosis venosa profunda, infección, rigidez o hemartrosis.

\section{Resultados radiológicos}

Al analizar las radiografías mediante la clasificación de Ahlbäck en los pacientes afectos de SVP, no se observaron cambios artrósicos significativos al comparar las radiografías preoperatorias con las del final del seguimiento, ni en los casos de SVPL ni en los casos de SVPD $(p=0,001)$

\section{Recidivas de la enfermedad}

Ocho de los 24 pacientes (33\%) presentaron una recidiva, con un tiempo medio entre la primera intervención y la recidiva de la SVP de 56,9 meses (rango, 23 - 168 
meses). Todos los casos correspondían a la forma difusa de la SVP. A 2 de ellos se les realizó radioterapia externa, a 5 se les volvió a efectuar una resección artroscópica y a otro caso se le realizó una cirugía abierta por vía de acceso posterior (vía de Trickey) para acabar de resecar el tejido enfermo que afectaba el espacio extraarticular del hueco poplíteo. Los pacientes de este grupo se encontraban hasta el último control asintomáticos clínica y radiológicamente tras un seguimiento mínimo de 21 meses después de la recidiva. 


\section{DISCUSIÓN}

El principal hallazgo de este estudio fue que tras una única resección artroscópica en la SVP localizada se obtienen buenos resultados funcionales y un estado de curación a medio plazo. Sin embargo, el porcentaje de pacientes que presentan recidivas de la enfermedad en la forma difusa es considerablemente alto. De todas maneras, la asociación en estos casos de una segunda resección artroscópica o algún otro procedimiento coadyuvante permitió controlar la enfermedad al menos a corto plazo en el $87,5 \%$ de los pacientes. De forma similar, De Ponti et al ${ }^{8}$, observaron que tras un seguimiento medio de 5 años, el riesgo de recurrencia era del $20 \%$ en SVPD tratadas mediante la técnica artroscópica sin otro tratamiento adyuvante. Por su parte, Zvijac et al ${ }^{9}$ reportaron un índice de recurrencia de la SVPD de un 14\%, en pacientes tratados artroscópicamente tras una media de 3,5 años de seguimiento.

Por otro lado, en un estudio de Colman et $\mathrm{al}^{19}$ donde estudiaron el riesgo de recurrencia de la SVPD con diferentes técnicas, observaron una menor recurrencia con la técnica mixta (artroscópica/abierta) en comparación con la artroscópica o abierta aislada: $9 \%$ vs $62 \%$ vs $64 \%$, respectivamente. Flandry et al ${ }^{4}$, describieron su serie con la técnica abierta mediante múltiples abordajes, donde sólo encontraron dos recurrencias en un grupo de 23 pacientes con un seguimiento medio de 58 meses.

Se han descrito déficits de movilidad postoperatoria. En los estudios de Johansson et $\mathrm{al}^{5} \mathrm{y}$ de Flandry et $\mathrm{al}^{4}$ se observaron una limitación del rango de movilidad de la 
rodilla intervenida mediante técnica abierta, con una media de 0-15ํㅡㄹ de extensión y de $90-130^{\circ}$ de flexión, en un $24 \%$ y $50 \%$ de los pacientes, respectivamente. Por el contrario, en el presente estudio se observó que tras la resección con técnica artroscópica se obtuvo una extensión completa en todos los casos y una flexión media de $127^{\circ}$.

Desde el punto de vista de las escalas funcionales, se observó una mejoría en las escalas indistintamente de si se trataba de una SVPD o SVPF. Gu et $a^{20}$ compararon SVPD tratadas artroscópicamente versus cirugía abierta y observaron una mejoría del IKDC desde unos 56,3 puntos de media preoperatorios hasta 89,4 puntos a los 3 años de la cirugía en los tratados artroscópicamente $(p<0,001)$ y desde unos 58,2 puntos de media en el preoperatorio hasta unos 72,1 puntos a los 3 años de la cirugía en los tratados mediante técnica abierta $(p<0,001)$. En la presente investigación también observamos una mejoría funcional en dicha escala que pasó de 47,8 a 78,4 puntos. De forma similar, en el estudio de Auregan et al ${ }^{21}$ evidenciaron buenos resultados funcionales y niveles de actividad con mejoras significativas sin encontrar tampoco diferencias entre SVPL y SVPD.

Una de las peores consecuencias de la SVP son los cambios degenerativos precoces en pacientes por otro lado sanos y jóvenes. Flandy et al ${ }^{4}$ reportaron un $48 \%$ de cambios degenerativos avanzados posterior a la realización de una sinovectomía abierta. Durante el seguimiento de los pacientes incluidos en el presente estudio no se objetivó ningún caso con artrosis avanzada secundaria a la intervención. Esto podría atribuirse a la técnica artroscópica, que es obviamente menos invasiva y agresiva. 
La principal limitación del presente trabajo radica en ser un estudio retrospectivo observacional con una muestra de pacientes limitada y sin disponer de un grupo control a los que se les hubiera realizado cirugía abierta, con una mediana de 60 meses de seguimiento.

\section{CONCLUSIÓN}

La SVP resecada mediante técnica artroscópica presenta buenos resultados funcionales y curativos a medio plazo con una baja morbilidad. La forma difusa de la SVP requiere con frecuencia una segunda intervención quirúrgica por su alta tasa de recidiva tras su resección artroscópica. 


\section{BIBLIOGRAFÍA}

1. Nakahara H, Matsuda S, Harimaya K, Sakamoto A, Matsumoto Y, Okazaki K, et al. Clinical results of open synovectomy for treatment of diffuse pigmented villonodular synovitis of the knee: case series and review of literature. Knee. 2012;19(5):684-7.

2. Tyler WK, Vidal AF, Williams RJ, Healey JH. Pigmented villonodular synovitis. J Am Acad Orthop Surg. 2006;14(6):376-85.

3. Sharma V, Cheng EY. Outcomes after excision of pigmented villonodular synovitis of the knee. Clin Orthop Relat Res. 2009;467(11):2852-8.

4. Flandry FC, Hughston JC, Jacobson KE, Barrack RL, McCann SB, Kurtz DM. Surgical treatment of diffuse pigmented villonodular synovitis of the knee. Clin Orthop Relat Res 1994;300:183-92.

5. Johansson JE, Ajjoub S, Coughlin LP, Wener JA, Cruess RL. Pigmented villonodular synovitis of joints. Clin Orthop Relat Res. 1982;163:159-66.

6. Chin KR, Barr SJ, Winalski C, Zurakowski D, Brick GW. Treatment of advanced primary and recurrent diffuse pigmented villonodular synovitis of the knee. J Bone Joint Surg Am. 2002;84-A(12):2192-202. 
7. Ogilvie-Harris DJ, McLean J, Zarnett ME. Pigmented villonodular synovitis of the knee. The results of total arthroscopic synovectomy, partial, arthroscopic synovectomy, and arthroscopic local excision. Bone Joint Surg Am. 1992;74(1):11923.

8. De Ponti A, Sansone V, Malcherè M. Result of arthroscopic treatment of pigmented villonodular synovitis of the knee. Arthroscopy. 2003;19(6):602-7.

9. Zvijac JE, Lau AC, Hechtman KS, Uribe JW, Tjin-A-Tsoi EW. Arthroscopic treatment of pigmented villonodular synovitis of the knee. Arthroscopy. 1999;15:613617

10. O'Sullivan B, Cummings B, Catton C, Bell R, Davis A, Fornasier V, Goldberg R. Outcome following radiation treatment for high-risk pigmented villonodular synovitis. Int J Radiation Oncology Biol Phys.1995;32:777-86.

11. Ottaviani S, Ayral X, Dougados M, Gossec L. Pigmented villonodular synovitis: a retrospective single-center study of 122 cases and review of the literature. Semin Arthritis Rheum. 2011;40(6):539-46.

12. Carpintero P, Garcia MA, Gascón E, Serrano J, Mesa M. Sinovitis villonodular pigmentada difusa. Revisión de 13 casos. Rev Esp Cir Osteoart. 1998; 33: 161-5.

13. Sanchis V, Baixauli E, Monteagudo JC, Fernández CI. Sinovitis villonodular pigmentada (forma difusa). Rev Esp de Cir Ost 1989;24:23-6. 
14. Valenti JR, Cara JA, Mora G. Sinovitis villonodular pigmentada de localitzación en la extremidad inferior. Rev Ortop Traum 1995;39:26-9.

15. Irrgang JJ, Anderson AF, Boland AL et al. Development and validation of the International Knee Documentation Committee Subjective Knee Form. Am J Sports Med 2001;29:600-613.

16. Kirkley A, Griffin A, Whelan D. The Development and Validation of a Quality of Life-Measurement Tool for Patients With Meniscal Pathology: The Western Ontario Meniscal Evaluation Tool (WOMET). Clin J Sport Med 2007;17:349-356.

17. Kujala UM, Jaakkola LH, Koskinen SK, Taimela S, Hurme M, Nelimarkka O. Scoring of patellofemoral disorders. Arthroscopy 1993;9:159-163.

18. Tegner Y, Lysholm J. Rating systems in the evaluation of knee ligament injuries. Clin Orthop Relat Res 1985; 198:43-9.

19. Colman MW, Ye J, Weiss KR, Goodman MA, McGough RL 3rd. Does combined open and arthroscopic synovectomy for diffuse PVNS of the knee improve recurrence rates? Clin Orthop Relat Res. 2013;471(3):883-90.

20. Gu HF, Zhang SJ, Zhao C, Chen Y, BiQ. Comparison of open and arthroscopic surgery for treatment of diffuse pigmented villonodular synovitis of the knee. Knee Surg Sports Traumatol Arthrosc. 2014;22(11):2830-6. 
21. Aurégan JC, Bohu Y, Lefevre N, Klouche S, Naouri JF, Herman S, Hardy P. Primary arthroscopic synovectomy for pigmented villo-nodular synovitis of the knee: recurrence rate and functional outcomes after a mean followup of seven years. Orthop Traumatol Surg Res. 2013;99(8):937-43. 
Tabla 1. Resultados demográficos.

\begin{tabular}{|l|l|}
\hline Edad (años) & 37 (rango, 13-62) \\
\hline Sexo (varón/mujer) & $13 / 11$ \\
\hline Lateralidad (derecha/izquierda) & $12 / 12$ \\
\hline SVP & \\
$\quad$ - Local & 11 pacientes \\
- Difusa & 13 pacientes \\
\hline SVP con lesiones asociadas & 6 pacientes \\
\hline
\end{tabular}


Tabla 2. Resultados de los test en el preoperatorio y en el postoperatorio.

\begin{tabular}{|l|l|l|l|}
\hline TEST & PREOPERATORIO & POSTOPOPERATORIO & P (VALOR) $^{\star}$ \\
\hline IKDC & $47,8(24,1-65)$ & $78,4(36,8-100)$ & 0,001 \\
\hline WOMET & $59,2(41,3-78)$ & $21,8(0,1-53,9)$ & 0,001 \\
\hline KUJALA & $51,57(35-79)$ & $85,6(60-100)$ & 0,001 \\
\hline TEGNER & $1,92(1-3)$ & $3(1-7)$ & 0,042 \\
\hline
\end{tabular}

* p significativa $<0,005$. 
Tabla 3. Resultados de los test en el preoperatorio, en el postoperatorio y la media de ellos en SVPL y SVPD.

\begin{tabular}{|c|c|c|c|c|c|c|c|c|c|}
\hline \multirow{2}{*}{ TEST } & \multicolumn{2}{|c|}{ PREOPERATORIO } & \multirow{2}{*}{ P (VALOR) ${ }^{*}$} & \multicolumn{2}{|c|}{ POSTOPERATORIO } & \multirow{2}{*}{$\begin{array}{l}\text { P } \\
\text { (VALOR) }^{*}\end{array}$} & \multicolumn{2}{|c|}{ DIFERENCIA } & \multirow{2}{*}{$\begin{array}{l}\text { P } \\
\text { (VALOR)* }^{*}\end{array}$} \\
\hline & SVPL & SVPD & & SVPL & SVPD & & SVPL & SVPD & \\
\hline IKDC & $\begin{array}{l}36,8 \\
(28,5-58,8)\end{array}$ & $\begin{array}{l}47,7 \\
(24,1-65)\end{array}$ & 0,571 & $\begin{array}{l}87,7 \\
(55,2-100)\end{array}$ & $\begin{array}{l}74,7 \\
(36,8-100)\end{array}$ & 0,151 & $\begin{array}{l}38,6 \\
(12,1-49)\end{array}$ & $\begin{array}{l}27 \\
(8,4-45)\end{array}$ & 0,83 \\
\hline WOMET & $\begin{array}{l}60,7(41,3- \\
77,5)\end{array}$ & $\begin{array}{l}58,8(43,3 \\
-78)\end{array}$ & 0,573 & $\begin{array}{l}34,8(0,1- \\
47,1)\end{array}$ & $\begin{array}{l}30,4(1,5 \text { - } \\
53,9)\end{array}$ & 0,852 & \begin{tabular}{|l|}
74,1 \\
$(51,3-97,6)$
\end{tabular} & $\begin{array}{l}75,4(53,5 \\
-98,8)\end{array}$ & 0,892 \\
\hline KUJALA & $\begin{array}{l}49,8 \\
(35-58,8)\end{array}$ & $\begin{array}{l}53,58 \\
(39-79)\end{array}$ & 1 & $\begin{array}{l}90,3 \\
(61-100)\end{array}$ & $\begin{array}{l}83,6 \\
(60-100)\end{array}$ & 0,151 & $\begin{array}{l}40,5 \\
(11-52)\end{array}$ & $\begin{array}{l}30 \\
(7-54) \\
\end{array}$ & 0,125 \\
\hline TEGNER & $1,7(1-2)$ & $2(1-3)$ & 0,683 & $3,7(1-7)$ & $2,62(1-6)$ & 0,461 & $2(1-5)$ & $1,6(1-3)$ & 0,214 \\
\hline
\end{tabular}

* $p$ significativa $<0,005$. 
Tabla 4. Resultados medios de los test comparando pacientes sólo con SVP versus pacientes con lesiones asociadas.

\begin{tabular}{|l|l|l|l|}
\hline TEST & $\mathbf{1 8 / 2 4}($ SVP) & $\begin{array}{l}\text { 6/24 (SVP + } \\
\text { LESIONES } \\
\text { ASOCIADAS) }\end{array}$ & $\mathbf{P}$ (VALOR) $^{\star}$ \\
\hline KUJALA & $28,47(12-43)$ & $46,8(44,2-49,5)$ & 0,026 \\
\hline IKDC & $27,2(12,4-40,6)$ & $40,6(33,2-46,5)$ & 0,05 \\
\hline WOMET & $35,4(13,3-51,1)$ & $44(38-51,8)$ & 0,33 \\
\hline
\end{tabular}

* $p$ significativa $<0,005$. 
Figura (Figure)
Click here to download high resolution image

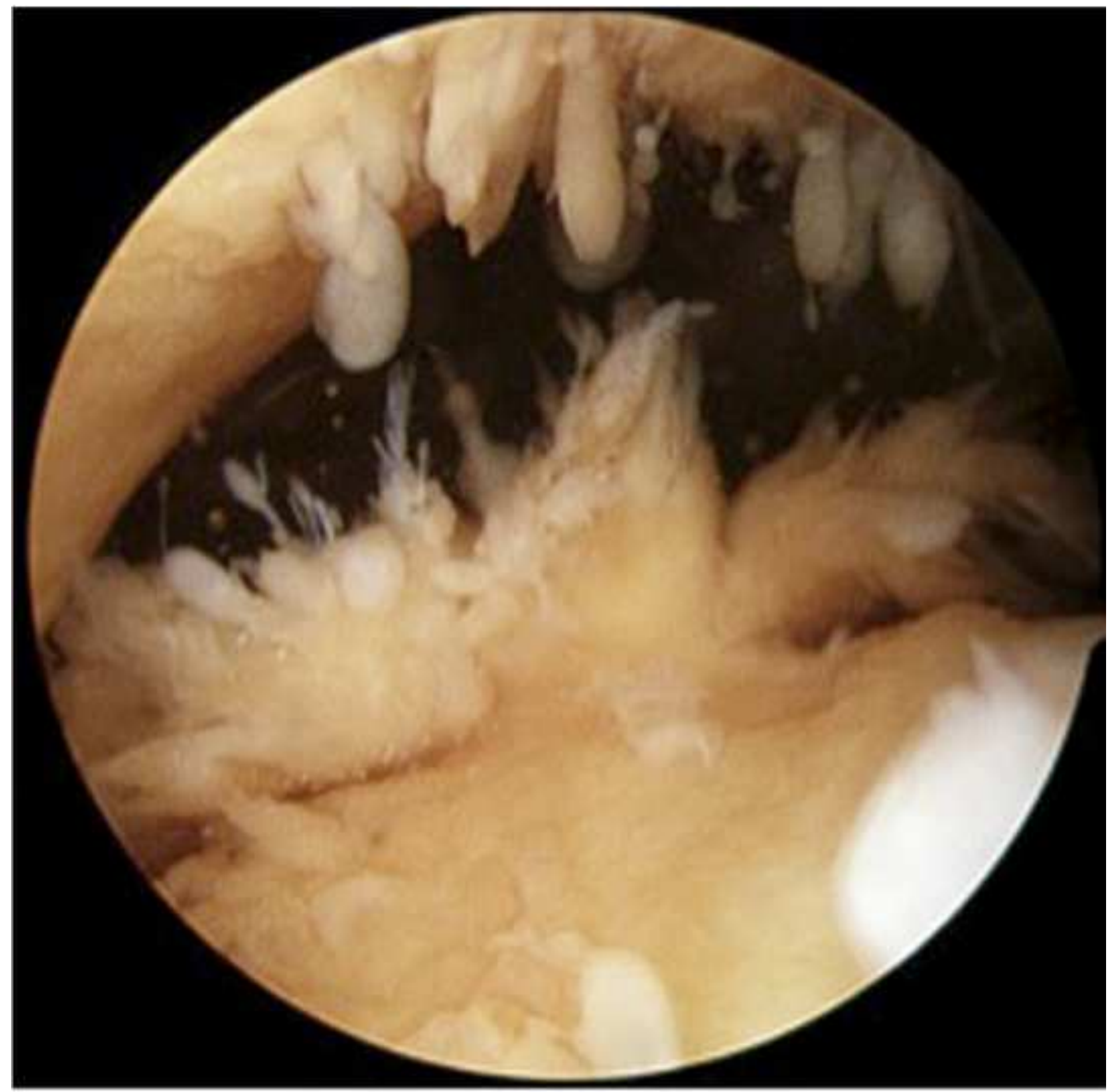




\section{Figura (Figure)}

Click here to download high resolution image

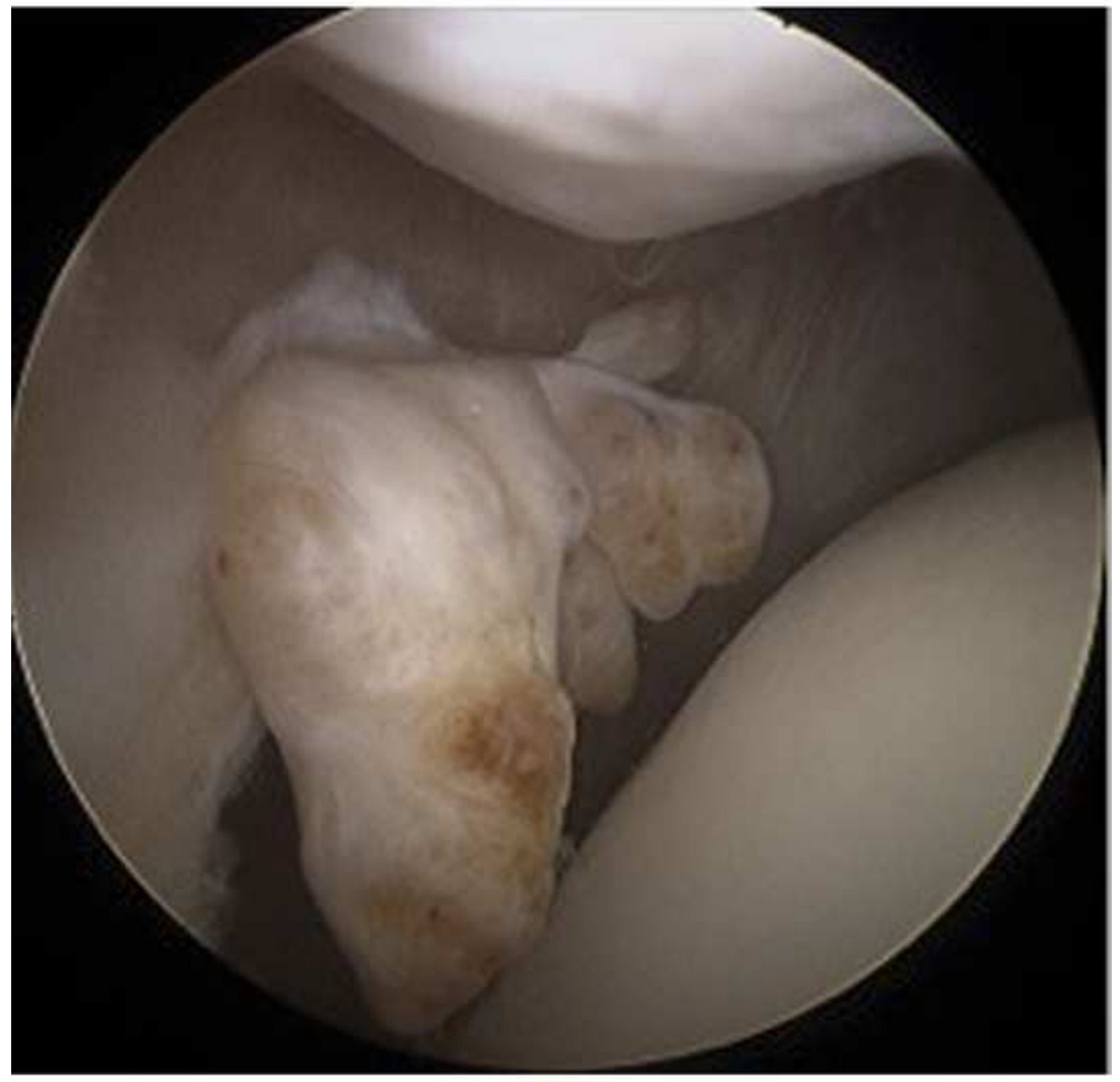


Figura (Figure)
Click here to download high resolution image

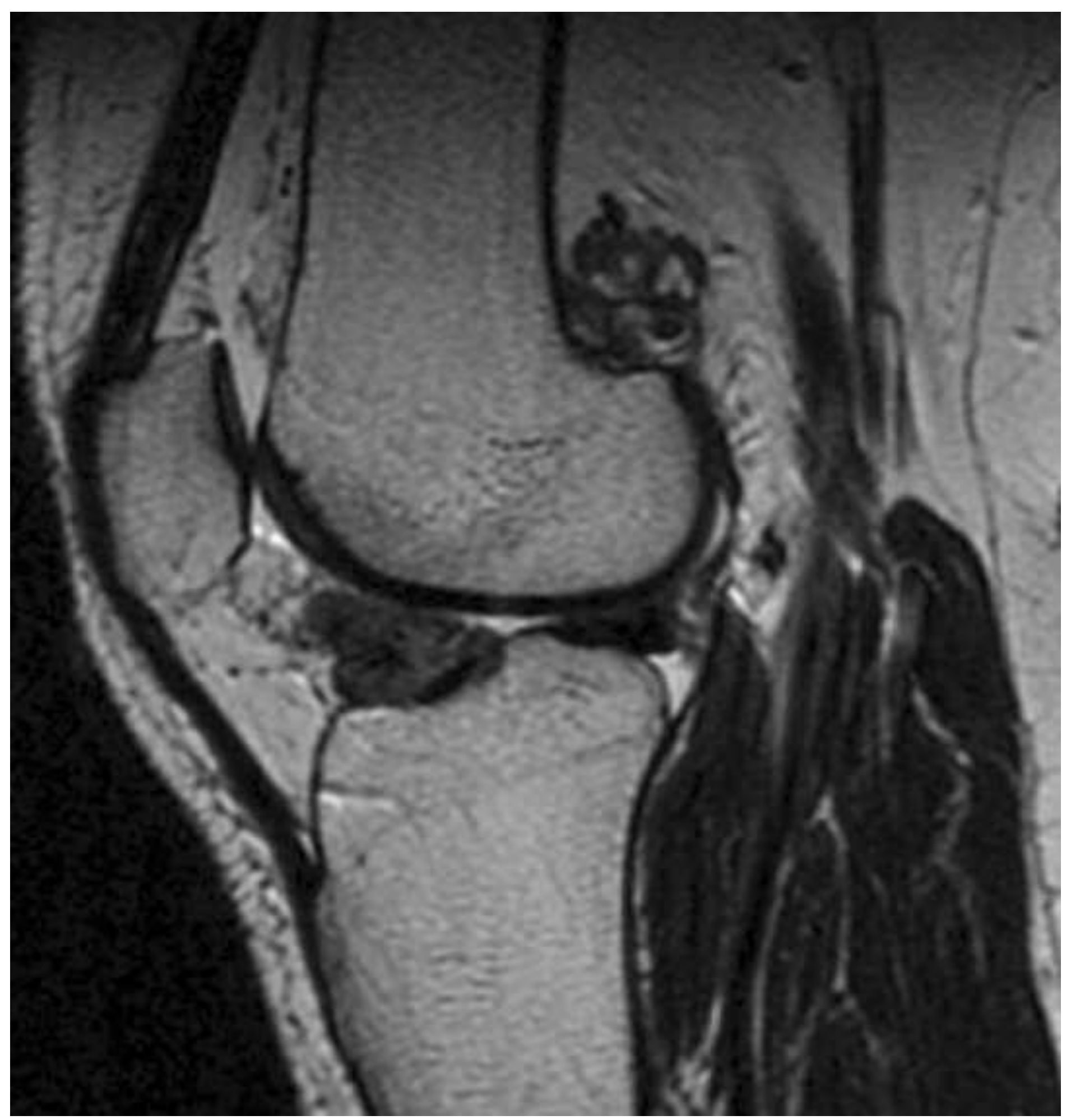


